\title{
Fill-level prediction in online valley-filling algorithms for electric vehicle charging
}

\author{
Martijn H. H. Schoot Uiterkamp, Marco E. T. Gerards, and Johann L. Hurink \\ Faculty of Electrical Engineering, Mathematics and Computer Science \\ University of Twente, Enschede, the Netherlands \\ Email: \{m.h.h.schootuiterkamp, m.e.t.gerards, j.1.hurink\}@utwente.nl
}

\begin{abstract}
Due to the large increase in electric vehicles (EVs), smart charging strategies are required in order for the distribution grid to accommodate all these EVs. Many charging strategies either assume that future loads are known in advance, or use predictions of these loads as input. However, accurate prediction of uncontrollable load is very difficult. Online valleyfilling algorithms circumvent this problem by determining the charging profile based on a prediction of the fill-level: a single parameter that characterizes the optimal EV schedule. This paper presents a simple, but accurate, method to predict this fill-level. We show that near-optimal charging profiles with an optimality gap of less than $1 \%$ can be realized when our method is used to predict the input level for the online valley-filling approach. Furthermore, our method is very fast and thus suitable for use in decentralized energy management systems that employ the online valley-filling approach.
\end{abstract}

Index Terms - electric vehicle charging, valley-filling, online algorithm, prediction

\section{INTRODUCTION}

The number of electric vehicles (EVs) in the world increases rapidly and has already passed three million in 2017 [1]. This increase of EVs leads to a large increase in loads in residential distribution grids. Field tests have shown that uncontrolled charging of EVs can lead to very high peak consumption and even outages (e.g., [2]). Therefore, smart control of EV charging is required to preserve a proper operation of the grid. In particular, peak loads should be minimized in order to prevent overloading of the grid and reduce losses.

As input to compute the optimal charging profile, many EV charging strategies use the uncontrollable load (or base load) of a household. In such strategies, it is often assumed that one knows this future base load in advance. However, in practice, such knowledge is not available. As a consequence, predictions of the base loads have to be used as input. Many methods exist for predicting the base load profile [3]. However, already small errors in a prediction, especially with regard to the time of large demand peaks, can lead to bad solutions when the EV charging is determined based on this prediction. Therefore, much research focuses on solution methods that take the uncertainty of future base loads into account. Examples of such methods are robust optimization (e.g., [4]), stochastic programming (e.g., [5]) and multi-agent systems (e.g, [6]).

A widely used concept for scheduling EV charging is valleyfilling. Valley-filling aims to obtain a flat energy profile by "filling" valleys in the base load profile with EV charging. In this way, the EV is not charged during high peaks of the uncontrollable demand and, as a consequence, does not increase the maximum peak consumption. Several works apply valley-filling as a subroutine in decentralized EV fleet control strategies (e.g., [7]-[11]). Often, such approaches aim at finding charging profiles that are optimal to the valley-filling objective by iteratively updating the charging profiles of individual EVs. However, few works exploit the fact that the optimal charging profile can be characterized by a single parameter. This parameter, which is called the fill-level, is the level up to which the valleys in the base load profile are filled with EV charge. Some studies (e.g., [12]-[14]) take the optimal fill-level (the fill-level that corresponds to the optimal charging profile) or an approximation of this level actively into account when computing the charging profile. This results in very good online charging strategies that deliver near-optimal solutions. Furthermore, it can be shown that these strategies are very robust against unforeseen behavior of the base load.

This paper presents a simple, but accurate, method to predict the fill-level that is required as input for online valley-filling algorithms. We show that our method is able to predict the optimal fill-level to such a high accuracy that an online solution can be obtained with an optimality gap of less than $1 \%$. The method also predicts the fill-level accurately for cases with high PV production peaks, provided that the amount that has to be charged is large enough to compensate for the resulting production peaks. The required user input for our method is a risk parameter that represents a preference for either under- or over-predicting the optimal fill-level. We argue that this preference is influenced by the context wherein the EV charging is managed and thus should be taken into account when predicting fill-levels. Our method uses information of historical days to compute the optimal charging profile and fill-level for charging sessions similar to the current one. In this way, we can learn the behavior of the optimal fill-level for the current charging session. We show that the resulting behavior is very stable, which enables us to compute very accurate predictions.

The remainder of this paper is organized as follows. In Section II, we explain both the offline and online valley-filling concepts in more detail. Section III presents our approach to predict the fill-level, which we validate in Section IV in a simulation study. Finally, Section V provides our conclusions. 


\section{THE VALLEY-FILLING APPROACH}

This section describes the valley-filling approach in more detail. Section II-A introduces the EV charging problem and discusses the offline valley-filling approach and Section II-B reviews the online version. The latter is basically a reformulation of the method described in [14].

\section{A. The offline valley-filling approach}

We first introduce the EV charging problem that we consider in this paper. We assume that the charging window (the time window during which the EV can be charged) is given. We divide this period into $T$ time intervals $\mathcal{T}=\{1, \ldots, T\}$. The variable $x_{t}$ now denotes the energy volume that is charged during time interval $t$ and $p_{t}$ denotes the base load at interval $t$. We call $\vec{x}=\left(x_{t}\right)_{t \in \mathcal{T}}$ and $\vec{p}=\left(p_{t}\right)_{t \in \mathcal{T}}$ the charging profile and base load profile respectively. We assume that the required charge, i.e., the amount that needs to be charged within the charging window, is known on forehand and denote this charge by $C$. Furthermore, we denote by $\bar{x}$ the maximum charging power of the EV for each time interval. The objective is to obtain a 'proper' combined base load and EV profile, which is generally specified as 'as flat as possible'. This can be modeled by minimizing the 2 -norm of $\vec{p}+\vec{x}$. All together, we obtain the following optimization problem:

$$
\begin{aligned}
\text { ChargeEV }: \min _{\vec{x}} \sqrt{\sum_{t=1}^{T}\left(p_{t}+x_{t}\right)^{2}} \\
\text { s.t. } \sum_{t=1}^{T} x_{t}=C, \\
0 \leq x_{t} \leq \bar{x}, \quad t=1, \ldots, T .
\end{aligned}
$$

Charge EV is a separable quadratic resource allocation problem for which many solution methods exist [15]. The valleyfilling approach is one of them and is basically a breakpoint search or multiplier search method. The general idea behind such methods is that a single parameter uniquely characterizes an optimal solution. In terms of ChargeEV and the common way to represent valley-filling approaches in the EV charging literature, this characterization states that there exists a scalar $Z$ called the fill-level such that, for any optimal solution $\vec{x}^{*}$ to ChargeEV, we have

$$
x_{t}^{*}= \begin{cases}0 & \text { if } p_{t}>Z \\ \bar{x} & \text { if } p_{t}+\bar{x}<Z, \\ Z-p_{t} & \text { otherwise }\end{cases}
$$

or, shortly,

$$
x_{t}^{*}=\max \left(0, \min \left(Z-p_{t}, \bar{x}\right)\right) .
$$

Note this implies that computing this optimal $Z$ solves ChargeEV.

Figure 1 illustrates the valley-filling approach. For this example, $\bar{x}$ is large enough such that $p_{t}+x_{t}=Z$ for any interval $t$ whose base load does not exceed $Z$.

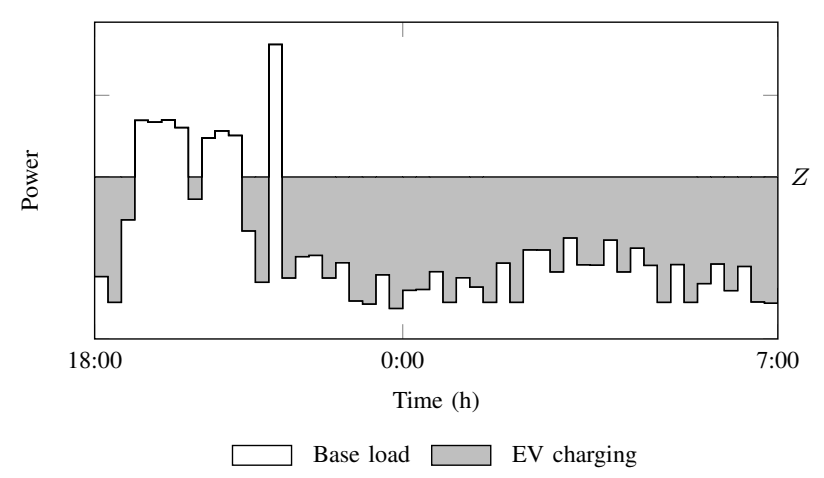

Fig. 1. EV charging using the optimal fill-level $Z$. The EV starts charging at 18:00h and its charging deadline is 7:00h.

\section{B. The online valley-filling approach}

In the offline setting, only $Z$ is unknown on forehand. However, in the online setting, also $\vec{p}$ is unknown. One approach is to use predictions of $\vec{p}$ as input. However, predicting the base load of a household proves to be very difficult in practice (see, e.g., [16]). Fortunately, we can use (1) to obtain a charging strategy that only requires a prediction of $Z$. In Section III, we show that $Z$ is much easier to predict than $\vec{p}$.

For now, suppose we have a prediction $\hat{Z}$ of $Z$. For each time interval $t$, we now determine the charge $\hat{x}_{t}$ as follows:

$$
\hat{x}_{t}=\max \left(0, \min \left(\hat{Z}-p_{t}, \bar{x}\right)\right) .
$$

Employing this decision rule to compute an online charging strategy has three big advantages over other methods. First, we do not require a prediction of the complete base load profile $\vec{p}$ at the start of the first time interval. Instead, we only require a measurement or prediction of $p_{t}$ at the start of interval $t$ itself. Second, even if the prediction $\hat{Z}$ of $Z$ is not very good, the resulting charging profile is still flat for most of the time window due to the nature of the decision rule (2). Third, this method it robust against variations in both the magnitude and time of consumption peaks. Regardless of when such a peak occurs, the decision rule (2) ensures that no or only little charging is done at that moment.

Algorithm 1 captures the described online approach for a given time interval $t$. Here, $\tilde{C}$ is the amount that has already been charged until the start of interval $t$, i.e., $\tilde{C}=\sum_{i=1}^{t-1} \hat{x}_{i}$. Apart from the decision rule (2) in Line 1, Algorithm 1 contains two additional rules to correct for the error in $\hat{Z}$. If $\hat{Z}>Z$, the charging requirement is met before the end of the time window. Line 2 ensures that, in this case, the EV only charges the remainder of the charging requirement or remains idle in these intervals (see Fig. 2a). On the other hand, if $\hat{Z}<Z$, the $\mathrm{EV}$ does not meet its charging requirement before the end of the time window if (2) is always used. As a consequence, from a certain interval on, charging at maximum power is needed (see Lines 3-5) to ensure that the EV is charged in time (see Fig. 2b). 

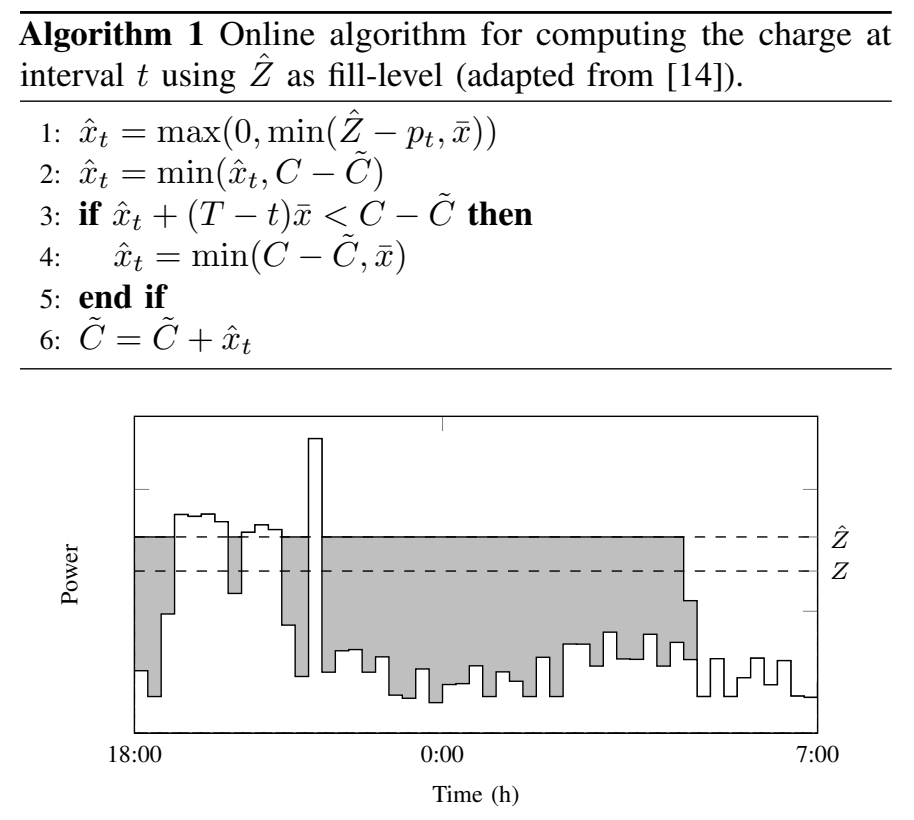

$\square$ Base load $\square$ EV charging under $\hat{Z}$

(a) $\hat{Z}>Z$

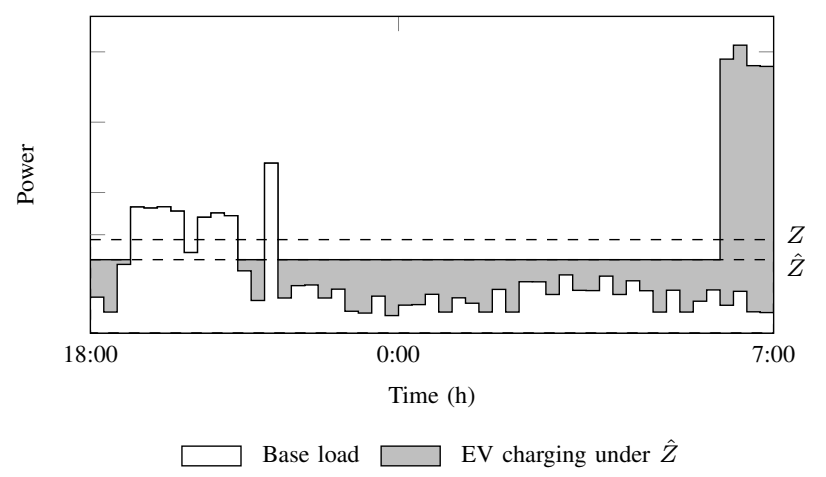

(b) $\hat{Z}<Z$

Fig. 2. EV charging using a fill-level $\hat{Z}$ with (a) $\hat{Z}>Z$ and (b) $\hat{Z}<Z$.

\section{PREDICTION APPROACH}

Algorithm 1 needs a prediction $\hat{Z}$ of the optimal fill-level $Z$ as input. This section is devoted to deriving a prediction approach for such $\hat{Z}$. In Section III-A, we discuss the impacts of under- and over-predicting $Z$ and how this should be taken into account when predicting $\hat{Z}$. Based on this analysis, we propose to model $Z$ as a stochastic variable with a fixed, but unknown, probability distribution. In Section III-B, we explain how we obtain an approximation to this distribution.

\section{A. Under- and over-predicting $Z$}

There are several reasons why either under- or overpredicting $Z$ could be preferable in a given situation. In both cases, as argued in Section II-B, the combined base load and $\mathrm{EV}$ profile is flattened for the main part of the charging window. On the one hand, when $\hat{Z}<Z$, this flattened part of the profile is lower than for $\hat{Z}>Z$, but in this case, a large peak that can last for several intervals occurs near the end of the period. On the other hand, when $\hat{Z}>Z$, no EV peaks near the end are created, but the flattened part of the profile is higher. Depending on the context wherein the online valley-filling approach is applied, one of these outcomes might be preferred.

In earlier work [14], [17], we aimed to predict $Z$ such that the objective value of Charge $E V$ is minimized and argued that this objective value grows more rapidly for $\hat{Z}<Z$ than for $\hat{Z}>Z$. As a consequence, when we aim to flatten the combined profile as much as possible when charging a single EV, overpredicting the level is the preferred option. However, when we look from a neighborhood perspective, under-predicting $Z$ for a few of the EVs can be beneficial for the aggregated power profile. To see this, suppose multiple EVs within the same neighborhood are charging simultaneously and their individual charging is managed by means of the online valley-filling approach. Also suppose that they have a similar charging window and deadline (e.g., during the night and deadlines between 7:00h and 9:00h). If the fill-levels of all EVs are overpredicted, then all EVs will be finished charging before their actual deadline. Thus, near the end of the charging window, almost no charging is done by any of the EVs. Therefore, it is not that harmful if the fill-levels of a few of the EVs are underpredicted: this simply means that a few EVs will have to charge at full power near the end of the period. As a consequence, they fill up the valley near the end of the charging window and in addition decrease the aggregated power consumption during the main part of their charging window.

We conclude that any preference for either under- or overprediction of the optimal fill-level should be taken actively into account when developing a prediction approach. We choose to model this preference by means of a risk parameter $\alpha \in[0,1]$, where $\alpha=0$ implies that we always want $\hat{Z} \leq Z$ and $\alpha=1$ implies that we always want $\hat{Z} \geq Z$. Our goal is then to compute a prediction $\hat{Z}$ such that the probability that $\hat{Z} \geq Z$ is $\alpha$. Suppose that $Z$ is a stochastic variable that has a cumulative distribution function (CDF) $F_{Z}$. Then the goal stated above corresponds to finding $\hat{Z}$ such that

$$
\alpha=\mathbb{P}(\hat{Z} \geq Z)=F_{Z}(\hat{Z}),
$$

which is equivalent to

$$
\hat{Z}=F_{Z}^{-1}(\alpha) .
$$

This yields a simple approach for predicting $Z$. Given $F_{Z}^{-1}$ and $\alpha$, we can simply compute $\hat{Z}$ by applying (3). However, as $F_{Z}$, and thus $F_{Z}^{-1}$ are unknown, we propose to approximate $F_{Z}$ by an appropriate distribution. In Section III-B, we analyze the behavior of $Z$ in order to choose such a suitable approximation.

\section{B. Computing the distribution of $Z$}

Section II-A showed that the value of $Z$ depends on $\vec{p}, C$ and $\bar{x}$. Of these parameters, $\vec{p}$ is the only unknown in the online setting. By using base load profiles of previous days as input for Charge $E V$ while leaving the other parameters $(C$ and $\bar{x}$ ) and the charging window unchanged, we can learn the behavior of $Z$. More precisely, by this we get a set of samples of $Z$ that we can use to learn the distribution of $Z$. Note that 


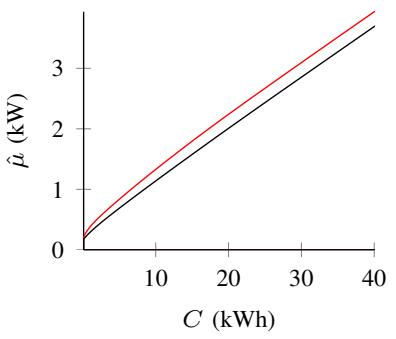

(a) Sample mean $(\hat{\mu})$

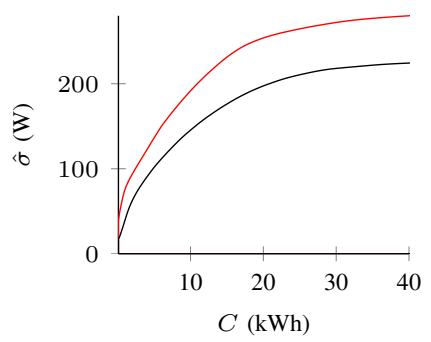

(b) Sample deviation $(\hat{\sigma})$
Fig. 3. The observed statistics of $Z$ for the charging windows 7:00h-19:00h (red) and 19:00h-7:00h (black).

we assume that the base load profile is independent of the base load profile of other days to keep the analysis and derivations in this subsection simple.

To observe the behavior of $Z$, we compute a sample set of $Z$-values for different values of $C$ using base load data from 100 previous days for a single household located in southern Germany. We have chosen not to assess the influence of $\bar{x}$ since in practice, $\bar{x}$ is generally large enough to ensure that no charging at maximum power is required in the optimal solution and thus the influence of $\bar{x}$ on $Z$ is negligible. Figure 3 shows the corresponding sample means $\hat{\mu}$ and sample deviations $\hat{\sigma}$ for two different charging windows. Figures $3 \mathrm{a}$ implies that $\hat{\mu}$ is proportional to $C$. This implies that as $C$ increases, $Z$ depends more on $C$ and less on the base load profile. Figures $3 b$ indicates that $\hat{\sigma}$ becomes almost constant when $C$ increases. Together with the proportionality of $\hat{\mu}$, this implies that the values of $Z$ hardly differ relative to each other for large $C$. This suggests that $Z$ varies only slightly over the course of multiple days, which greatly increases its predictability.

The above analysis suggests that $Z$ can be modeled accurately as a stochastic variable. To determine $F_{Z}$, we do not assume $Z$ to follow a specific distribution. Instead, we construct its empirical CDF $F_{Z}^{\mathrm{emp}}$ from the samples of $Z$ and use this function as a first approximation of the $\mathrm{CDF}$ of $Z$. One disadvantage of using an empirical CDF to compute $\hat{Z}$ is that it is a discontinuous function, meaning that there exist values of $\alpha$ for which $\left(F_{Z}^{\mathrm{emp}}\right)^{-1}$ is undefined. Therefore, in order to compute $\hat{Z}$ using (3), we construct from $F_{Z}^{\text {emp }}$ a piecewise linear approximation that has the discontinuities of $F_{Z}^{\text {emp }}$ as its breakpoints. The resulting CDF, which we denote by $\hat{F}_{Z}$, is continuous and thus its inverse $\left(\hat{F}_{Z}\right)^{-1}$ exists and can be used to evaluate (3).

\section{Simulation STUDY}

In this section, we validate the prediction approach presented in Section III and evaluate its efficiency. For this, we simulate the charging of a single EV using the online valley-filling approach, where we predict $\hat{Z}$ using our approach. Section IV-A explains the setup of the simulations and the different scenarios that we consider. Section IV-B presents and discusses the simulation results. All simulations and computations in this

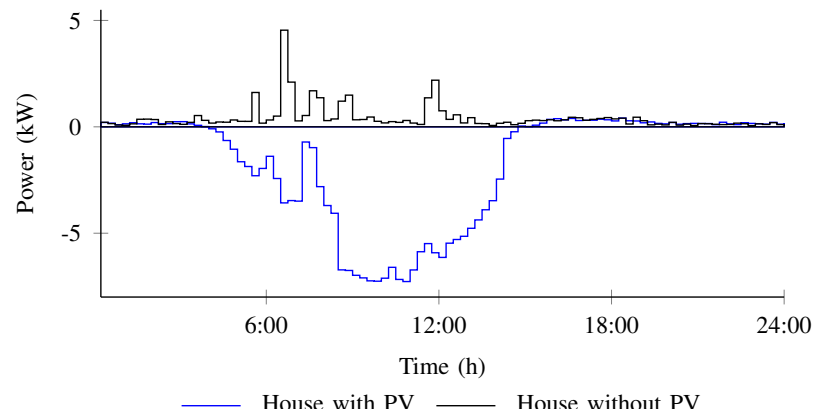

Fig. 4. Typical base load profile of the two houses used in the PV study.

section are executed on a Dell Inspiron 15 with an Intel Core i7-6700HQ CPU at $2.60 \mathrm{GHz}$ and $16 \mathrm{~GB}$ of RAM.

\section{A. Setup and scenarios}

For all simulations, we divide the charging windows into 15 minute time intervals. We consider two different charging windows that represent charging during the day and charging during the night, namely 7:00h-19:00h and 19:00h-7:00h respectively. We use the Nissan Leaf 2018 model as a reference $\mathrm{EV}$, which means that the considered battery capacity is $40 \mathrm{kWh}$ [18]. In order to assess the impact of different charging requirements, we consider both the situation where the battery is empty and needs to be fully charged $(C=40 \mathrm{kWh})$ and the situation where only $25 \%$ of the capacity needs to be charged $(C=10 \mathrm{kWh})$. We assume that a home charger with a maximum rate of $6.6 \mathrm{~kW}$ is used. We use the base load profile of the same household as the one that we considered in Section III-B. To construct $\left(\hat{F}_{Z}\right)^{-1}$, we consider four different sets of previous days as training data. These sets consist of the previous 3, 10, 50 and 100 days respectively. To compute the sample set of optimal fill-levels, i.e., to solve ChargeEV for these previous days, we apply the EV charging algorithm in [19]. This algorithm is very fast in practice and thus significantly reduces the computation time of the prediction approach. For each of the above scenarios, we calculate the predicted fill-level $\hat{Z}$ using (3) for a broad range of risk parameters $A=\{0.05,0.15, \ldots, 0.95\}$. Subsequently, we carry out the online valley-filling approach, i.e., Algorithm 1, using this $\hat{Z}$ as input fill-level. We repeat this procedure for 100 subsequent days.

In a second step, we assess the influence of local PV production on our approach, we carry out our approach on two similar households, both also located in southern Germany, of which one has PV panels and thus a large production peak during the day. Figure 4 shows a typical daily base load profile for these two houses. We now consider only the charging window 7:00h-19:00h and 10 previous days as sample days, and repeat the approach for 10 subsequent days. Since $C=40 \mathrm{kWh}$ is too small to flatten the base load profile of the house with PV, we also simulated these cases for an EV with a larger battery, where we used a Tesla model S as reference EV $(C=100 \mathrm{kWh}$, $\bar{x}=11.5 \mathrm{~kW}$ ) [20]. 
TABLE I

FRACTION OF CASES WHERE $\hat{Z}>Z$. VALUES CLOSE TO $\alpha$ IMPLY AN ACCURATE MODELING OF THE RISK SPECIFIED BY $\alpha$.

\begin{tabular}{|c|c|c|c|c|c|c|c|c|c|c|c|c|c|c|c|c|}
\hline \multirow[b]{3}{*}{$\alpha$} & \multicolumn{8}{|c|}{ 7:00h-19:00h } & \multicolumn{8}{|c|}{ 19:00h-7:00h } \\
\hline & \multicolumn{4}{|c|}{$C=10 \mathrm{kWh}$} & \multicolumn{4}{|c|}{$C=40 \mathrm{kWh}$} & \multicolumn{4}{|c|}{$C=10 \mathrm{kWh}$} & \multicolumn{4}{|c|}{$C=40 \mathrm{kWh}$} \\
\hline & $3 \mathrm{~d}$ & $10 \mathrm{~d}$ & $50 \mathrm{~d}$ & $100 \mathrm{~d}$ & $3 d$ & $10 \mathrm{~d}$ & $50 \mathrm{~d}$ & $100 \mathrm{~d}$ & $3 \mathrm{~d}$ & $10 \mathrm{~d}$ & $50 \mathrm{~d}$ & $100 \mathrm{~d}$ & $3 \mathrm{~d}$ & $10 \mathrm{~d}$ & $50 \mathrm{~d}$ & $100 \mathrm{~d}$ \\
\hline 0.05 & 0.28 & 0.13 & 0.15 & 0.18 & 0.25 & 0.14 & 0.15 & 0.15 & 0.31 & 0.11 & 0.12 & 0.18 & 0.30 & 0.11 & 0.11 & 0.18 \\
\hline 0.15 & 0.33 & 0.19 & 0.25 & 0.31 & 0.34 & 0.22 & 0.22 & 0.27 & 0.35 & 0.21 & 0.24 & 0.31 & 0.37 & 0.21 & 0.24 & 0.31 \\
\hline 0.25 & 0.39 & 0.30 & 0.34 & 0.43 & 0.41 & 0.28 & 0.37 & 0.41 & 0.41 & 0.31 & 0.29 & 0.35 & 0.44 & 0.31 & 0.29 & 0.34 \\
\hline 0.35 & 0.47 & 0.41 & 0.47 & 0.53 & 0.46 & 0.37 & 0.45 & 0.48 & 0.46 & 0.43 & 0.35 & 0.42 & 0.47 & 0.42 & 0.35 & 0.40 \\
\hline 0.45 & 0.49 & 0.48 & 0.60 & 0.61 & 0.51 & 0.50 & 0.53 & 0.55 & 0.48 & 0.48 & 0.46 & 0.56 & 0.50 & 0.48 & 0.43 & 0.51 \\
\hline 0.55 & 0.57 & 0.58 & 0.65 & 0.70 & 0.56 & 0.58 & 0.66 & 0.70 & 0.57 & 0.54 & 0.60 & 0.66 & 0.58 & 0.54 & 0.55 & 0.64 \\
\hline 0.65 & 0.62 & 0.66 & 0.72 & 0.79 & 0.60 & 0.65 & 0.75 & 0.78 & 0.63 & 0.63 & 0.69 & 0.71 & 0.64 & 0.66 & 0.66 & 0.71 \\
\hline 0.75 & 0.66 & 0.73 & 0.81 & 0.83 & 0.67 & 0.73 & 0.80 & 0.83 & 0.64 & 0.73 & 0.83 & 0.85 & 0.66 & 0.72 & 0.80 & 0.83 \\
\hline 0.85 & 0.67 & 0.81 & 0.91 & 0.93 & 0.69 & 0.82 & 0.90 & 0.90 & 0.66 & 0.81 & 0.92 & 0.94 & 0.68 & 0.80 & 0.90 & 0.90 \\
\hline 0.95 & 0.71 & 0.88 & 0.96 & 0.96 & 0.71 & 0.89 & 0.95 & 0.96 & 0.71 & 0.92 & 0.98 & 0.98 & 0.70 & 0.92 & 0.96 & 0.97 \\
\hline
\end{tabular}

TABLE II

MEdian RATIO BETWEEN ONLINE AND OPTIMAL OBJECTIVE VALUE.

\begin{tabular}{|c|c|c|c|c|c|c|c|c|c|c|c|c|c|c|c|c|}
\hline \multirow[b]{3}{*}{$\alpha$} & \multicolumn{8}{|c|}{$7: 00 \mathrm{~h}-19: 00 \mathrm{~h}$} & \multicolumn{8}{|c|}{$19: 00 \mathrm{~h}-7: 00 \mathrm{~h}$} \\
\hline & \multicolumn{4}{|c|}{$C=10 \mathrm{kWh}$} & \multicolumn{4}{|c|}{$C=40 \mathrm{kWh}$} & \multicolumn{4}{|c|}{$C=10 \mathrm{kWh}$} & \multicolumn{4}{|c|}{$C=40 \mathrm{kWh}$} \\
\hline & $3 d$ & $10 \mathrm{~d}$ & $50 \mathrm{~d}$ & $100 \mathrm{~d}$ & $3 d$ & $10 \mathrm{~d}$ & $50 \mathrm{~d}$ & $100 \mathrm{~d}$ & $3 d$ & $10 \mathrm{~d}$ & $50 \mathrm{~d}$ & $100 \mathrm{~d}$ & $3 d$ & $10 \mathrm{~d}$ & $50 \mathrm{~d}$ & $100 \mathrm{~d}$ \\
\hline 0.05 & 1.101 & 1.181 & 1.171 & 1.162 & 1.022 & 1.021 & 1.021 & 1.019 & 1.054 & 1.074 & 1.123 & 1.093 & 1.009 & 1.009 & 1.010 & 1.009 \\
\hline 0.15 & 1.083 & 1.113 & 1.117 & 1.069 & 1.021 & 1.017 & 1.015 & 1.016 & 1.038 & 1.042 & 1.067 & 1.034 & 1.010 & 1.007 & 1.009 & 1.008 \\
\hline 0.25 & 1.074 & 1.071 & 1.057 & 1.057 & 1.020 & 1.019 & 1.018 & 1.016 & 1.036 & 1.030 & 1.039 & 1.028 & 1.012 & 1.005 & 1.008 & 1.008 \\
\hline 0.35 & 1.069 & 1.054 & 1.054 & 1.054 & 1.020 & 1.018 & 1.016 & 1.016 & 1.045 & 1.028 & 1.033 & 1.032 & 1.014 & 1.008 & 1.009 & 1.008 \\
\hline 0.45 & 1.066 & 1.055 & 1.055 & 1.056 & 1.020 & 1.017 & 1.019 & 1.020 & 1.050 & 1.029 & 1.038 & 1.031 & 1.015 & 1.009 & 1.010 & 1.010 \\
\hline 0.55 & 1.058 & 1.055 & 1.057 & 1.060 & 1.022 & 1.020 & 1.022 & 1.025 & 1.055 & 1.034 & 1.038 & 1.045 & 1.016 & 1.010 & 1.012 & 1.015 \\
\hline 0.65 & 1.066 & 1.064 & 1.063 & 1.065 & 1.026 & 1.025 & 1.025 & 1.032 & 1.057 & 1.037 & 1.046 & 1.051 & 1.021 & 1.016 & 1.018 & 1.022 \\
\hline 0.75 & 1.085 & 1.072 & 1.074 & 1.075 & 1.031 & 1.029 & 1.034 & 1.041 & 1.057 & 1.053 & 1.062 & 1.063 & 1.022 & 1.023 & 1.027 & 1.032 \\
\hline 0.85 & 1.08 & 1.091 & 1.095 & 1.108 & 1.032 & 1.041 & 1.056 & 1.061 & 1.061 & 1.061 & 1.081 & 1.087 & 1.025 & 1.029 & 1.042 & 1.048 \\
\hline 0.95 & 1.089 & 1.108 & 1.136 & 1.146 & 1.035 & 1.063 & 1.080 & 1.085 & 1.061 & 1.076 & 1.114 & 1.109 & 1.028 & 1.052 & 1.066 & 1.070 \\
\hline
\end{tabular}

\section{B. Results}

For each predicted level $\hat{Z}$, we determine whether $\hat{Z}$ is either an under- or over-prediction of the optimal level $Z$. Table I shows the ratio of days where $\hat{Z} \geq Z$ for each scenario and risk parameter in $A$. When this ratio is close to its corresponding $\alpha$, our approach is able to appropriately take into account the preference $\alpha$ for under- or over-prediction.

The charging requirements do not seem to influence the ratios significantly, However, in contrast, for the cases where 10 or 50 days are used as training days, the ratios tend to be closer to $\alpha$ than for the cases where 3 or 100 days are used. One possible explanation for this is that the previous 10 and 50 days represent both short-term and long-term behavior of $Z$. Information on both behaviors is required to make a good prediction. The previous 3 days clearly cannot provide us with information on the long-term behavior of $Z$. Similarly, if we use the previous 100 days and treat all these days equally, i.e., do not assign larger weights to recent days, we neglect any short-term behavior. For the cases that use 10 or 50 days, the ratios are close to $\alpha$ if $\alpha$ is not too small.

Table II shows the median ratio between the objective value of ChargeEV for the online solution and for the optimal solution. The number of sample days does not seem to significantly affect the ratios. In contrast, the online solutions for $C=40 \mathrm{kWh}$ are much better than those for $C=10 \mathrm{kWh}$ and in some cases even have an optimality gap of less
TABLE III

AVERAGE RUNNING TIME (S) OF THE PREDICTION APPROACH.

\begin{tabular}{l|cccc}
\hline Charging & \multicolumn{4}{|c}{ Day set size } \\
period & $3 \mathrm{~d}$ & 10d & $50 \mathrm{~d}$ & $100 \mathrm{~d}$ \\
\hline 7:00h-19:00h & 0.0034 & 0.0065 & 0.0266 & 0.0515 \\
19:00h-7:00h & 0.0029 & 0.0064 & 0.0262 & 0.0512 \\
\hline
\end{tabular}

than $1 \%$. This confirms our observations in Section III-B that the deviation of $Z$ compared to its mean decreases when $C$ increases. Moreover, the online solutions are better for $\alpha>0.5$ than for $\alpha<0.5$. This confirms the observations in [14] and [17] that, in general, predicting $Z$ too high leads to a better objective value than predicting $Z$ too low.

Table III shows the average execution time of the prediction approach. For each prediction, such an execution consists of the computation of the samples of $Z$, establishing $\left(\hat{F}_{Z}\right)^{-1}$ and computing $\hat{Z}$ using (3). We distinguished between the different charging windows and sample set sizes since these parameters influence the performance of our approach the most. The results in Table III indicate that, even when using 100 previous days as sample size, our prediction approach is very efficient and can thus be effectively used as a subroutine in, e.g., decentralized EV charging algorithms.

Finally, Table IV shows the results of the PV study. These results indicate that $\mathrm{PV}$ production diminishes the effectiveness 
TABLE IV

MEDian RATIO BETWEEN ONLINE AND OPTIMAL OBJECTIVE VALUE FOR THE PV STUDY.

\begin{tabular}{c|cc|cc|cc}
\hline & \multicolumn{2}{|c|}{$C=10 \mathrm{kWh}$} & \multicolumn{2}{c|}{$C=40 \mathrm{kWh}$} & \multicolumn{2}{c}{ Tesla case } \\
$\alpha$ & PV & No PV & PV & No PV & PV & No PV \\
\hline 0.05 & 2.373 & 1.096 & 1.718 & 1.010 & 1.087 & 1.002 \\
0.15 & 2.251 & 1.082 & 1.496 & 1.007 & 1.079 & 1.001 \\
0.25 & 2.073 & 1.061 & 1.440 & 1.008 & 1.056 & 1.001 \\
0.35 & 1.967 & 1.035 & 1.394 & 1.009 & 1.052 & 1.002 \\
0.45 & 1.796 & 1.025 & 1.318 & 1.009 & 1.045 & 1.002 \\
0.55 & 1.540 & 1.026 & 1.303 & 1.010 & 1.037 & 1.002 \\
0.65 & 1.254 & 1.028 & 1.186 & 1.009 & 1.038 & 1.002 \\
0.75 & 1.299 & 1.032 & 1.195 & 1.010 & 1.049 & 1.003 \\
0.85 & 1.324 & 1.027 & 1.196 & 1.011 & 1.057 & 1.003 \\
0.95 & 1.338 & 1.027 & 1.248 & 1.012 & 1.071 & 1.004 \\
\hline
\end{tabular}

of our prediction approach. However, the ratios improve, i.e., decrease as $C$ increases and for the Tesla case, the ratios for the house with PV are comparable with the results for $C=10 \mathrm{kWh}$ in Table II. This is in line with the observations in Section III-B on the deviation and predictability of $Z$.

\section{CONCLUSIONS}

In this paper, we considered EV charging using an online valley-filling approach and proposed a prediction method for the fill-level that is required as input for this approach. We showed that this level can be modeled accurately as a stochastic variable whose deviation converges to a constant value as the charging requirement increases. In addition, we carried out a simulation study that confirms these findings and shows that online valley-filling algorithms can deliver solutions with an optimality gap of less than $1 \%$ when the fill-level is predicted using our approach. This also holds when PV production is taken into account, as long as the amount that the EV has to charge is large enough to compensate for the resulting production peaks.

An important question for future research is how one can choose the risk parameter such that the online solution is close to the optimal solution. In addition, we plan to apply our prediction method to steer the charging of multiple EVs simultaneously using online valley-filling. For this, we plan to take into account both local and global valley-filling objectives by choosing suitable values for the risk parameter of each EV.

The predictability of the fill-level decreases significantly when PV production is present. Including weather forecasts in the prediction and careful selection of the used historical data might improve the predictability of PV production and thus of the fill-level. Therefore, future research focuses on adjusting our prediction approach to take these factors into account.

In this paper, we assumed that EVs have continuous charging rates. However, the theory and techniques presented in this paper can be applied directly to the case where the rates are binary, i.e., that EVs can only charge at a fixed rate. Earlier work [17] already explored online valley-filling approaches for this type of EVs. Difficulties arise, however, when an EV can choose from a finite number of charging rates since optimal solutions to those problems cannot be characterized anymore by a single fill-level. Future work thus focuses on developing online charging strategies for this type of EVs.

\section{ACKNOWLEDGMENT}

This research is conducted within the SIMPS project (647.002.003) supported by NWO and Eneco.

\section{REFERENCES}

[1] International Energy Agency, "Global EV Outlook 2018: Towards crossmodal electrification." [Online]. Available: https://webstore.iea.org/globalev-outlook-2018. Retrieved on July 18, 2018.

[2] G. Hoogsteen, A. Molderink, J. L. Hurink, G. J. M. Smit, B. Kootstra, and F. Schuring, "Charging electric vehicles, baking pizzas, and melting a fuse in Lochem," CIRED - Open Access Proceedings Journal, vol. 2017, no. 1, pp. 1629-1633, 2017.

[3] T. Hong and S. Fan, "Probabilistic electric load forecasting: A tutorial review," International Journal of Forecasting, vol. 32, no. 3, pp. 914-938, 2016.

[4] A. O'Connell, D. Flynn, and A. Keane, "Rolling multi-period optimization to control electric vehicle charging in distribution networks," IEEE Transactions on Power Systems, vol. 29, no. 1, pp. 340-348, 2014.

[5] K. Clement-Nyns, E. Haesen, and J. Driesen, "The impact of charging plug-in hybrid electric vehicles on a residential distribution grid," IEEE Transactions on Power Systems, vol. 25, no. 1, pp. 371-380, 2010.

[6] E. L. Karfopoulos and N. D. Hatziargyriou, "A multi-agent system for controlled charging of a large population of electric vehicles," IEEE Transactions on Power Systems, vol. 28, no. 2, pp. 1196-1204, 2013.

[7] Z. Ma, D. S. Callaway, and I. A. Hiskens, "Decentralized charging control of large populations of plug-in electric vehicles," IEEE Transactions on Control Systems Technology, vol. 21, no. 1, pp. 67-78, 2013.

[8] L. Gan, U. Topcu, and S. H. Low, "Optimal decentralized protocol for electric vehicle charging," IEEE Transactions on Power Systems, vol. 28 , no. 2, pp. 940-951, 2013.

[9] K. Zhang, L. Xu, M. Ouyang, H. Wang, L. Lu, J. Li, and Z. Li, "Optimal decentralized valley-filling charging strategy for electric vehicles," Energy Conversion and Management, vol. 78, pp. 537-550, 2014.

[10] J. Rivera, C. Goebel, and H. A. Jacobsen, "Distributed convex optimization for electric vehicle aggregators," IEEE Transactions on Smart Grid, vol. 8, no. 4, pp. 1852-1863, 2017.

[11] L. Zhang, V. Kekatos, and G. B. Giannakis, "Scalable electric vehicle charging protocols," IEEE Transactions on Power Systems, vol. 32, no. 2, pp. 1451-1462, 2017.

[12] N. Chen, C. W. Tan, and T. Q. S. Quek, "Electric vehicle charging in smart grid: optimality and valley-filling algorithms," IEEE Journal of Selected Topics in Signal Processing, vol. 8, no. 6, pp. 1073-1083, 2014

[13] Y. Mou, H. Xing, Z. Lin, and M. Fu, "Decentralized optimal demand-side management for PHEV charging in a smart grid," IEEE Transactions on Smart Grid, vol. 6, no. 2, pp. 726-736, 2015.

[14] M. E. T. Gerards and J. L. Hurink, "Robust peak-shaving for a neighborhood with electric vehicles," Energies, vol. 9, no. 8(594), 2016

[15] M. Patriksson and C. Strömberg, "Algorithms for the continuous nonlinear resource allocation problem - new implementations and numerical studies," European Journal of Operational Research, vol. 243, no. 3, pp. 703-722, 2015.

[16] F. Javed, N. Arshad, F. Wallin, I. Vassileva, and E. Dahlquist, "Forecasting for demand response in smart grids: an analysis on use of anthropologic and structural data and short term multiple loads forecasting," Applied Energy, vol. 96, pp. 150-160, 2012.

[17] M. H. H. Schoot Uiterkamp, "Robust planning of electric vehicle charging," M.Sc. thesis, University of Twente, 2016.

[18] "New Nissan Leaf prices and specifications." [Online]. Available: https://www.nissan.co.uk/vehicles/new-vehicles/leaf/pricesspecifications.html. Retrieved on July 18, 2018.

[19] T. van der Klauw, M. E. T. Gerards, G. J. M. Smit, and J. L. Hurink, "Optimal scheduling of electrical vehicle charging under two types of steering signals," in 2014 IEEE PES Innovative Smart Grid Technologies Conference Europe, Istanbul, 2014.

[20] "Tesla support: Model S specifications." [Online]. Available: https://www.tesla.com/support/model-s-specifications. Retrieved on July $18,2018$. 